\title{
ESTILOS DE APRENDIZAGEM DOS ESTUDANTES DA DISCIPLINA DE ENDODONTIA DE UMA UNIVERSIDADE PRIVADA NO SUL DO BRASIL
}

\author{
ESTILOS DE APRENDIZAJE DE ESTUDIANTES DE ENDODONCIA EN UNA \\ UNIVERSIDAD PRIVADA DEL SUR DE BRASIL
}

\author{
LEARNING STYLES OF ENDODONTICS STUDENTS AT A PRIVATE UNIVERSITY \\ IN SOUTHERN BRAZIL
}

\author{
Prescila Mota de Oliveira KUBLITSKI ${ }^{1}$ \\ Paulo Henrique TOMAZINHO ${ }^{2}$ \\ Flávia Sens Fagundes TOMAZINHO 3 \\ João Armando BRANCHER ${ }^{4}$ \\ Denise Piotto LEONARDI ${ }^{5}$ \\ Marilisa Carneiro Leão GABARDO ${ }^{6}$
}

RESUMO: Foram avaliados os estilos de aprendizagem dos estudantes de Endodontia de um curso de graduação em Odontologia, por meio do Índice de Estilos de Aprendizagem de Felder-Soloman. Participaram 144 estudantes, dos turnos matutino e noturno, entre maio e junho de 2016. Destes, $80,6 \%$ eram do gênero feminino e $68,5 \%$ do turno da manhã. A média de idade foi de 22,94 anos e a mediana de notas na disciplina foi de 6,52. Os estilos predominantes foram sensorial, visual, ativo e sequencial, respectivamente. A correlação entre idade e turno, e a intensidade de cada estilo de aprendizado não tiveram diferença estatisticamente significativa $(\mathrm{p}>0,05)$. As mulheres foram mais sensoriais e os homens mais visuais $(\mathrm{p}=0,010)$. Na correlação entre média anual e estilos, houve diferença significativa para o sensorial e o intuitivo $(\mathrm{p}<0,001)$. A maioria teve preferência mista entre os estilos de aprendizagem, independentemente do turno.

PALAVRAS-CHAVE: Aprendizagem. Endodontia. Ensino.

1 Universidade Positivo (UP), Curitiba - PR - Brasil. Doutoranda no Programa de Pós-Graduação em Odontologia. ORCID: https://orcid.org/0000-0002-3828-2328. E-mail: prescilamota@hotmail.com

${ }^{2}$ Meta Aprendizagem Treinamento e Editora Ltda., Curitiba - PR - Brasil. Fundador da Meta Aprendizagem. Membro do Grupo Técnico de Inovação do Ensino Superior do SEMESP e Grupo de Inovação do SINEPE. Doutorado em Odontologia (UP). ORCID: https://orcid.org/0000-0002-6436-5231. E-mail: paulohtomazinho@gmail.com

3 Universidade Positivo (UP), Curitiba - PR - Brasil. Professora no Programa de Pós-Graduação em Odontologia. Doutorado em Odontologia (UNAERP). ORCID: https://orcid.org/0000-0001-5553-6943. E-mail: flaviatomazinho@gmail.com

${ }^{4}$ Universidade Positivo (UP), Curitiba - PR - Brasil. Professor no Programa de Pós-Graduação em Odontologia. Doutorado em Ciências da Saúde (PUCPR). ORCID: https://orcid.org/0000-0002-8914-702X. E-mail: brancher.a@gmail.com

${ }^{5}$ Universidade Positivo (UP), Curitiba - PR - Brasil. Especialização em Ortodontia (UP). Doutorado em Endodontia (UNESP). ORCID: https://orcid.org/0000-0003-1686-1078. E-mail: dpleonardi@gmail.com

6 Universidade Positivo (UP), Curitiba - PR - Brasil. Professora no Programa de Pós-Graduação em Odontologia. Doutorado em Odontologia (PUCPR). ORCID: https://orcid.org/0000-0001-6832-8158. E-mail: marilisagabardo@gmail.com

RPGE- Revista on line de Política e Gestão Educacional, Araraquara, v. 25, n. 2, p. 1544-1557, maio/ago. 2021. e-ISSN: 1519-9029 DOI: https://doi.org/10.22633/rpge.v25i2.14698 
RESUMEN: Los estilos de aprendizaje de los estudiantes de Endodoncia de un curso de pregrado en Odontología se evaluaron utilizando el índice de estilos de aprendizaje de Felder-Soloman. Participaron 144 estudiantes, de los turnos matutino y nocturno, entre mayo y junio de 2016. De estos, el 80,6\% fueron mujeres y el 68,5\% del turno matutino. La edad promedio fue de 22,94 años y la nota media en la disciplina fue de 6,52. Los estilos predominantes fueron sensorial, visual, activo y secuencial, respectivamente. La correlación entre la edad y el turno, y la intensidad de cada estilo de aprendizaje no tuvo diferencias estadisticamente significativas ( $p>0.05$ ). Las mujeres eran más sensoriales y los hombres más visuales $(p=0,010)$. En la correlación entre el promedio anual y los estilos, hubo diferencia significativa para el sensorial y el intuitivo $(p<0,001)$. La mayoría tenía una preferencia mixta entre los estilos de aprendizaje, independientemente del cambio.

PALABRAS CLAVE: Aprendizaje. Endodoncia. Enseñanza.

ABSTRACT: The learning styles of Endodontics students from an undergraduate Dentistry course were evaluated using the Felder-Soloman Learning Styles Index. 144 students participated, from the morning and night shifts, between May and June 2016. Of these, 80.6\% were female and $68.5 \%$ of the morning shift. The average age was 22.94 years and the median grade in the discipline was 6.52. The predominant styles were sensory, visual, active and sequential, respectively. The correlation between age and shift, and the intensity of each learning style had no statistically significant difference $(p>0.05)$. Women were more sensory and men more visual $(p=0.010)$. In the correlation between the annual average and the styles, there was a significant difference for the sensory and the intuitive $(p<0.001)$. Most had a mixed preference between learning styles, regardless of the shift.

KEYWORDS: Learning. Endodontics. Teaching.

\section{Introdução}

O processo de aprendizagem deve ser criativo para que o estudante consiga se tornar reflexivo e desenvolva outras habilidades intelectuais, de modo a aprender a construir o conhecimento, a ter capacidade de pensar a respeito dos assuntos e, sobretudo, a conseguir expor suas ideias. Desta forma, atualmente há necessidade e preocupação expressas pela busca de como os estudantes aprendem.

No caso de as atividades pedagógicas encontrarem-se centradas no estudante e nos resultados de aprendizagem, a identificação dos estilos de aprendizagem é um passo importante para a personalização do ensino. Os estilos se referem a preferências e a tendências individualizadas, o que influencia diretamente a maneira de aprender (ALMEIDA, 2010; SCHMITT; DOMINGUES, 2016). As características são particulares ao se adquirir conhecimentos, habilidades e atitudes, ou seja, cada indivíduo possui um estilo único e diferenciado no processo de sistematização do conhecimento (DA SILVA, 2006). Segundo 
Lopes (2002), derivam dos conceitos de tipologia e personalidade, e relacionam-se à forma particular de obter, reter, processar e organizar o conhecimento.

O conceito de estilo, em linguagem pedagógica, é utilizado para analisar vários comportamentos, ou seja, a forma como as pessoas atuam (ALONSO; GALLEGO; HONEY, 1999). Segundo Sadler-Smith (1997), a compreensão e o reconhecimento do conceito de estilo de aprendizagem são formas de ajudar as escolas a pensarem mais nos seus papéis e na organização cultural nos quais professores e alunos estão inseridos.

Em uma análise da produção nacional sobre habilidades sociais, Murta (2005), afirma que a formação das mesmas é definida por múltiplos aspectos, como movimentos verbais e não-verbais, cognitivo-afetivos, fisiológicos e aparência pessoal. Entretanto, na formação da habilidade social podem ocorrer falhas que ocasionam déficits expressivos, os quais estão associados ao fraco desempenho acadêmico (MURTA, 2005).

A atenção para as necessidades socioemocionais dos estudantes deve ser considerada para a construção de um ambiente educacional motivador, principalmente por parte dos professores, como pressupõem Guimarães e Boruchovitch (2004). Esses autores avaliaram a motivação dos alunos a partir do estilo motivacional dos professores, revelando ser esse um importante construto através do impacto positivo provocado no desempenho dos alunos.

À medida que o professor toma consciência da existência de diversos perfis, e que cada estudante tem sua própria maneira de aprender e de se relacionar, selecionando estratégias mais adequadas a um determinado tipo ou grupo de alunos, passa a promover um ensino orientado por esses parâmetros, com uso de estratégias que promovem um aprendizado mais eficaz e duradouro (KURI, 2004).

Sabe-se que o sistema educacional ainda é muito dependente do professor, da sala de aula e das técnicas e recursos instrucionais. O processo de ensino e aprendizagem se dá pela interação dos elementos de um ambiente educacional: instituição, professor, aluno e assunto (DA SILVA, 2006). Fritsch, Flores e Giraffa (2008) entendem que as estratégias de ensino devem incluir funções de orientação no desempenho das atividades, explanações dos fenômenos e processos, correções, bem como adaptações específicas e individuais, gerando desafios, explicações, exemplos e/ou contraexemplos no decorrer das interações.

Aqui, os estilos de aprendizagem vêm à tona, uma vez definidos como os diferentes modos de se executar um processo, já que o aprendizado em um sistema educacional estruturado envolve dois passos: recepção e processamento de informação, o que pode ser seguido sem uma ordem específica de realização (FELDER; SILVERMAN, 1988). Nesse contexto surge o Índice de Estilos de Aprendizagem - Index of Learning Styles (ILS) 
(FELDER; SOLOMAN, 1991), instrumento desenvolvido na Universidade Estadual da Carolina do Norte, que serve para determinar as preferências de aprendizagem em quatro dimensões do Modelo de Felder e Silverman (1988). O diferencial é que esse instrumento não contempla a dimensão indutiva/dedutiva do modelo anterior.

O ILS aborda, em cada dimensão, dois estilos opostos de aprendizagem: ativo/reflexivo, sensorial/intuitivo, visual/verbal e sequencial/global (VIOLA et al., 2007). O instrumento tem 44 questões com duas opções de escolha apenas (alternativa a ou b). Para cada uma das dimensões há 11 questões. A diferença entre os escores da dupla de cada dimensão indica qual é o estilo predominante ou preferido pelo respondente (FELDER; SOLOMAN, 1991).

No que diz respeito à confiabilidade e à validade do ILS, foram localizados trabalhos com resultados positivos em diversos países (FELDER; SPURLIN, 2005; HOSFORD; SIDERS, 2010; KU; SHEN, 2009), inclusive no Brasil (KURI, 2004; LOPES, 2002).

Com base no exposto, o presente estudo teve como objetivo descrever o perfil do estilo de aprendizagem de alunos da disciplina de Endodontia, do Curso de Graduação em Odontologia da Universidade Positivo, Curitiba, Brasil.

\section{Material e métodos}

Esse trabalho é um estudo transversal, exploratório e descritivo, que foi aprovado pelo Comitê de Ética em Pesquisa da Universidade Positivo, sob registro n. 2.045.917. Todos os sujeitos foram informados sobre o objetivo do estudo e assinaram o Termo de Consentimento Livre e Esclarecido. Para a escolha da amostra utilizou-se o método não probabilístico por conveniência, em que foram convidados a participar 179 estudantes do curso de graduação em Odontologia da Universidade Positivo, com idade igual ou superior a 18 anos. Foram incluídos aqueles que estavam regularmente matriculados na disciplina de Endodontia durante o período de maio a junho de 2016, de ambos os gêneros, e dos turnos matutino e noturno.

O estilo de aprendizagem foi avaliado por meio do ILS (FELDER; SILVERMAN, 1988) em versão validada para o português (LOPES, 2002). Esse instrumento é composto por 44 questões objetivas e identifica os estilos de aprendizagem dos estudantes, que podem ser: ativo/reflexivo, sensitivo/intuitivo, verbal/visual, indutivo/dedutivo e sequencial/global. Um link para acesso ao formulário eletrônico (Google Forms $\left.{ }^{\circledR}\right)$ foi disponibilizado aos estudantes.

Os dados foram tabulados e analisados em SPSS versão 21.0 (IBM Corp. Released 2012. IBM SPSS Statistics for Windows, Versão 21.0. Armonk, NY: IBM Corp.). Inicialmente 
foi feita análise descritiva e, em seguida, foram aplicados os testes de Mann-Whitney e de correlação de Spearman. Adotou-se o nível de significância 5\%.

\section{Resultados}

Participaram da pesquisa 144 estudantes (taxa de resposta $=78,2 \%$ ). O gênero feminino compôs $80,6 \%(n=116)$ da amostra; estudantes do turno na manhã representaram a maior proporção, com $n=98(68,5 \%)$. A média geral da idade foi de 22,94 anos (DP $\pm 4,67)$; a mediana das notas na disciplina foi 6,52, com mínimo de 0,60 e máximo de 8,60.

$\mathrm{Na}$ Tabela 1 são encontrados os valores medianos (com mínimo e máximo) das respostas dados aos estilos de aprendizagem avaliados.

Tabela 1 - Estilos de aprendizagem dos alunos da disciplina de Endodontia do Curso de Graduação em Odontologia da Universidade Positivo, Curitiba PR, Brasil (n = 144)

\begin{tabular}{cc}
\hline Estilos de aprendizagem & Mediana (mín.-máx.) \\
\hline Ativo & $6,48(1-11)$ \\
Reflexivo & $4,53(0-10)$ \\
Sensorial & $7,98(1-11)$ \\
Intuitivo & $3,05(0-10)$ \\
Visual & $7,25(1-11)$ \\
Verbal & $3,76(0-10)$ \\
Sequencial & $6,27(2-10)$ \\
Global & $4,73(1-9)$ \\
\hline
\end{tabular}

Fonte: Dados da pesquisa

Notou-se que o perfil dos estudantes foi composto, na maioria, pelos estilos sensorial, visual, ativo e sequencial, respectivamente. Observou-se, também, que entre as dimensões visual/verbal houve domínio do visual, ou seja, a maioria aproveita mais as imagens, sejam em explicações orais ou escritas (Tabela 1).

Quando em análise os turnos, houve predomínio pelos mesmos estilos: sensorial, visual, ativo e sequencial (Figuras 1 e 2). 
Figura 1 - Estilos de aprendizagem dos alunos do período matutino da disciplina de Endodontia do Curso de Graduação em Odontologia da Universidade Positivo, Curitiba PR, Brasil $(n=144)$

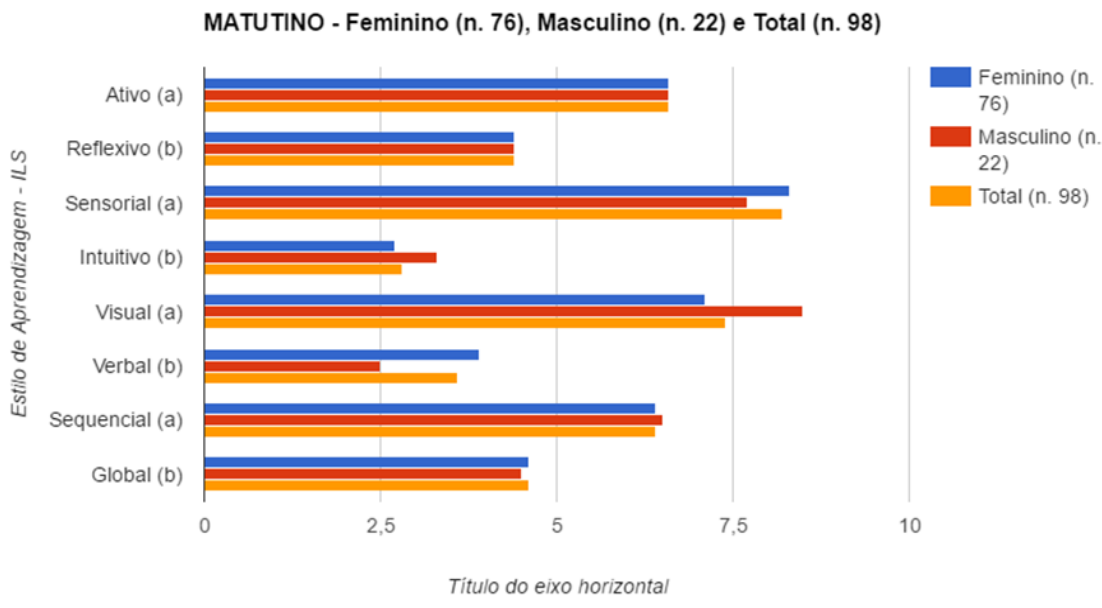

Fonte: Dados da pesquisa

Figura 2 - Estilos de aprendizagem dos alunos do período noturno da Disciplina de Endodontia do Curso de Graduação em Odontologia da Universidade Positivo, Curitiba PR, Brasil $(n=144)$

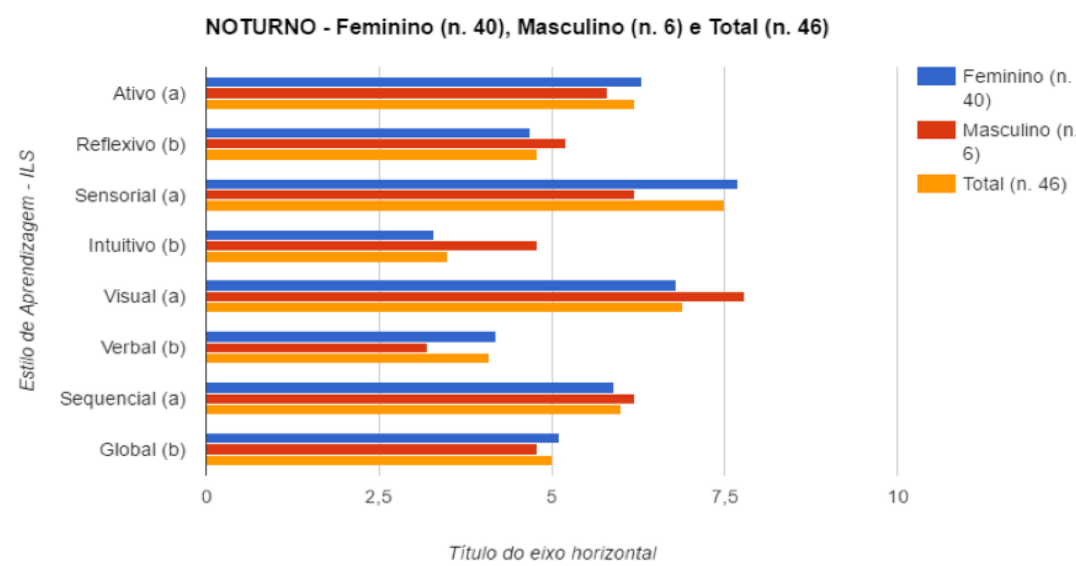

Fonte: Dados da pesquisa

A correlação entre a variável idade com a intensidade de cada estilo de aprendizado não revelou significância estatística $(\mathrm{p}>0,05)$.

Quanto à associação entre gênero e estilos de aprendizagem, os resultados estão descritos na Tabela 2. Independentemente do turno em que os alunos estão matriculados, destacou-se o fato que, de modo geral, mulheres são mais verbais e homens são mais visuais quanto aos seus estilos preferenciais (Tabela 2). 
Tabela 2 - Associação entre gênero e estilos de aprendizagem dos alunos do período matutino da Disciplina de Endodontia do Curso de Graduação em Odontologia da Universidade Positivo, Curitiba PR, Brasil $(\mathrm{n}=144)$

\begin{tabular}{lccc}
\hline $\begin{array}{c}\text { Estilos de } \\
\text { aprendizagem }\end{array}$ & $\begin{array}{c}\text { Feminino } \\
\text { mediana (mín.-máx.) }\end{array}$ & $\begin{array}{c}\text { Masculino } \\
\text { mediana (mín.-máx.) }\end{array}$ & $\begin{array}{c}\text { Valor de } \\
\mathbf{p}^{*}\end{array}$ \\
\hline Ativo & $7(3-10)$ & $6(1-11)$ & 0,663 \\
Reflexivo & $4(1-8)$ & $5(4-11)$ & 0,655 \\
Sensorial & $8(4-11)$ & $7(1-11)$ & 0,073 \\
Intuitivo & $3(0-7)$ & $4(0-10)$ & 0,071 \\
Visual & $7(1-11)$ & $9(4-11)$ & $\mathbf{0 , 0 1 0}$ \\
Verbal & $4(0-10)$ & $2(0-7)$ & $\mathbf{0 , 0 1 0}$ \\
Sequencial & $6(2-10)$ & $6(3-9)$ & 0,393 \\
Global & $5(1-9)$ & $5(2-8)$ & 0,391 \\
\hline
\end{tabular}

*Teste de Mann-Whitney

Nota: Valores em negrito são estatisticamente significativos

Fonte: Dados da pesquisa

No teste de associação de Mann-Whitney, quando avaliadas as variáveis turno e estilo de aprendizagem, também não foi encontrada significância $(p>0,05)$.

A análise de correlação entre a média anual dos alunos e a intensidade de cada estilo de aprendizagem pode ser vista na Tabela 3. Aqui se pode afirmar que houve uma correlação estatisticamente significativa entre a média anual e os estilos sensorial e intuitivo $(p<0,001)$.

Tabela 3 - Correlação entre média anual dos alunos e intensidade dos estilos de aprendizagem dos alunos do período matutino da disciplina de Endodontia do Curso de Graduação em Odontologia da Universidade Positivo, Curitiba PR, Brasil (n = 144)

\begin{tabular}{lcc}
\hline Estilos de aprendizagem & Valor de $\mathbf{p}^{*}$ & Rs \\
\hline Ativo & 0,387 & 0,740 \\
Reflexivo & 0,387 & 0,740 \\
Sensorial & $\mathbf{< 0 , 0 0 1}$ & 0,312 \\
Intuitivo & $<\mathbf{0 , 0 0 1}$ & 0,321 \\
Visual & 0,948 & 0,006 \\
Verbal & 0,948 & 0,006 \\
Sequencial & 0,150 & 0,122 \\
Global & 0,150 & 0,122 \\
\hline
\end{tabular}

*Teste de Mann-Whitney

Nota: Valores em negrito são estatisticamente significativos

Fonte: Dados da pesquisa

\section{Discussão}

Este estudo teve como objetivo analisar os diferentes estilos de aprendizagem entre estudantes da disciplina de Endodontia de um Curso de Graduação em Odontologia, onde foi revelada uma variação entre os estilos sensorial, visual, ativo e sequencial, 
independentemente de turno de aulas. No que diz respeito ao gênero, as mulheres adotam mais o estilo sensorial, enquanto os homens são mais visuais. Já a melhor média de notas anual foi obtida por estudantes que disseram adotar os estilos sensorial e intuitivo.

Certamente, cada estudante desenvolve estratégias e tem preferências quanto ao modo de receber, organizar e reter informações (DA SILVA, 2006). Tais estratégias podem, de maneira geral, ser chamadas de estilos de aprendizagem, que são definidos como a modalidade preferida pelos sujeitos para aprender (ALMEIDA, 2010; SCHMITT; DOMINGUES, 2016). Um estilo de aprendizagem não inviabiliza o outro; ao contrário, os estilos de aprendizagem podem se complementar. De fato, é bastante comum encontrar preferências de estilos entre os estudantes. Uma pesquisa realizada com indivíduos de diversas áreas, inclusive da Odontologia, revelou que os estilos predominantes encontrados, em ordem decrescente, foram: sensorial $(76,19 \%)$, sequencial $(67,0 \%)$, verbal $(57,7 \%)$ e ativo (56,7\%) (BIRRER; MINELLO, 2016).

Um estudo em que foram avaliadas as preferências de estilos de aprendizagem com estudantes do curso de Engenharia foi o de Carmo et al. (2010). Os autores concluíram que o estilo de aprendizagem da maioria dos pesquisados foi o ativo, o que pressupõe que a grade curricular e o processo de ensino e aprendizagem adotado pelos docentes promovem ou fortalecem tal fato. Estudantes que preferem o estilo ativo de aplicam conceitos na prática após discutirem os temas em grupos. Talvez essa seja uma característica inerente aos cursos em que há a necessidade de se pensar e elaborar projetos coletivamente.

Com estudantes de Contabilidade, pesquisas identificaram prevalência dos estilos na ordem: ativo, visual, sensorial e sequencial (DE SOUZA; AVELINO; TAKAMATSU, 2017; MENDES DA SILVA; DUTRA DE OLIVEIRA NETO, 2010). Por sua vez, em estudantes portugueses de Administração observou-se que são mais sensoriais (CARVALHO et al., 2019).

Na área da saúde, em pesquisa com 172 estudantes de Farmácia, a média de idade foi similar ao aqui encontrado, com valor de 21,1 anos e $75,4 \%$ eram mulheres. Quanto aos estilos de aprendizagem, predominou o sensorial (87,8\%), seguido do visual $(69,8 \%)$ e do sequencial $(61,6 \%)$, dados que corroboram os aqui encontrados nas duas primeiras posições (BECKER, 2013). Já no estudo piloto, também com estudantes de Farmácia, autores apontaram os mesmos resultados da presente pesquisa, com predomínio da percepção sensorial, da ativa e da sequencial (DE JESUS et al., 2017).

$\mathrm{Na}$ presente pesquisa, o estilo de aprendizagem prevalente foi o sensorial, entretanto, muitos estudantes preferiram os estilos visual, ativo e o sequencial. A prevalência do sensorial 
pode ser explicada pelo contexto de estudo e aprendizagem desses estudantes, que lidam com fatos e casos clínicos reais, já os sensoriais preferem informações percebidas através dos sentidos e tendem a ser metódicos (CURY, 2000).

É importante salientar que os estudantes avaliados nesta pesquisa estavam cursando a disciplina de Endodontia laboratorial, e a percepção tátil/sensorial de um instrumento endodôntico explorando um canal radicular é primordial para o sucesso da terapia endodôntica. Talvez, mais do que qualquer outra especialidade da Odontologia, o estilo de aprendizagem sensorial seja extremamente importante para o aprendizado da Endodontia.

Outro dado relevante é que o próprio perfil prático do curso de Odontologia exige uma capacidade perceptiva mais detalhista dos estudantes, que acabam sendo treinados para desenvolver sua capacidade sensorial. Isso, de fato, está descrito na dimensão sensorial da aprendizagem, que compreende aqueles que gostam de aprender com casos concretos, são mais detalhistas, memorizam fatos com facilidade, e saem-se bem em trabalhos práticos (FELDER; SPURLIN, 2005). Isso também foi constatado em pesquisa com estudantes do mesmo curso em fase pré-clínica de cirurgia, com mais respondentes também afirmando serem sensoriais (OMAR, 2017).

O segundo estilo de aprendizagem mais prevalente nos estudantes que participaram desta pesquisa foi o visual. Estudantes que optam por esse estilo preferem que as informações sejam repassadas a eles por meio de esquemas, fluxogramas, figuras, ilustrações, filmes ou quaisquer outros meios de representação visual (FELDER; FELDER; DIETZ, 2002). A respeito das peculiaridades de cada Curso, intuitivamente é plausível que estudantes de Odontologia se enquadrem melhor no estilo visual. Essa ideia não é nova e, em pesquisa anterior, realizada com estudantes de Ortodontia, constatou-se que estes são aprendizes altamente visuais, mas com preferência por estratégias de aprendizagem sequencial e sensorial (HUGHES et al., 2009). No presente estudo observou-se que na dimensão visual/verbal há domínio do estilo visual, ou seja, a maioria tira maior proveito das imagens, sejam em explicações orais ou escritas. Nesse sentido, o uso das explicações orais ou escritas pode ocorrer, mas não se deve deixar de incluir de imagens, diagramas, tabelas e gráficos durante as explicações, pois elas, por si só, não contribuirão tão efetivamente para a maioria (FELDER; SPURLIN, 2005).

$\mathrm{Na}$ dimensão ativo pode-se dizer que houve um equilíbrio entre os estudantes que compreendem melhor quando discutem ou explicam para outras pessoas. Por fim, a dimensão sequencial indica que os estudantes preferem caminhos lógicos, aprendem melhor os conteúdos apresentados de forma linear e encadeada (FELDER; SPURLIN, 2005).

RPGE- Revista on line de Política e Gestão Educacional, Araraquara, v. 25, n. 2, p. 1544-1557, maio/ago. 2021. e-ISSN: 1519-9029 
Nesta pesquisa, evidenciou-se também que as mulheres adotam um estilo de predominantemente verbal, fato também apontado em estudo realizado com graduandas do curso de Odontologia (LIMA, 2007). Uma explicação um pouco mais aprofundada para a predileção das mulheres pelo estilo verbal remete ao fato que, para a maioria delas, a comunicação verbal é essencial para a compreensão de fatos e para o desenvolvimento de um bom relacionamento interpessoal (WOOD, 1995). Desta forma, não é surpresa que mulheres apontem e adotem o estilo de aprendizagem verbal como principal forma de aprendizagem. Do ponto de vista pedagógico, estudantes verbais preferem ouvir as informações (CURY, 2000).

De maneira geral, qualquer processo de aprendizagem deve envolver quatro processos básicos: percepção, retenção, processamento e organização das informações (SILVA et al., 2012) e, nesta pesquisa, um dado relevante diz respeito à percepção da informação por parte dos estudantes e que envolve a dimensão sensorial e a dimensão intuitiva. Estudantes sensoriais e intuitivos foram aqueles que obtiveram melhores notas no período de realização da coleta de dados. As principais características observadas em estudantes sensoriais são o cuidado com os detalhes, o cuidado com o método em si; já os estudantes intuitivos vislumbram novas possibilidades e são, portanto, inovadores.

\section{Considerações finais}

Este estudo fornece evidências de que o processo de percepção da informação e a maneira como o estudante processa a mesma, assemelham-se muito ao estilo de aprendizagem que envolve as dimensões sensorial e intuitiva. Esse fato pode servir de subsídio para que professores pensem em estratégias e metodologias mais bem direcionadas.

Obviamente as abordagens pedagógicas e as diferenças individuais de cada estudante também vão influenciar no estilo de aprendizagem. Sob essa ótica, os professores devem utilizar estratégias compatíveis com as necessidades dos estudantes. Por outro lado, as diferenças individuais que são determinadas por uma série de fatores, entre eles os componentes afetivos, personalidade, as características emocionais relacionadas à persistência, responsabilidade, motivação e interação entre pares, devem ser cuidadosamente avaliadas. Com isso, é possível inferir que o conhecimento dos estilos de aprendizagem individuais e predominantes em uma turma pode trazer benefícios aos estudantes e professores. Aos estudantes que, ao tomarem consciência de como aprendem melhor, podem desenvolver mais essas capacidades e assim podem aprender mais, melhor e mais rápido; aos 
professores, uma vez conhecendo os estilos de aprendizagem predominantes em uma dada turma, podem adaptar seus processos de ensino e aprendizagem para assim coincidir com as características desta, mas sem negligenciar os múltiplos estilos existentes.

O instrumento, aqui empregado, foi capaz de aferir o estilo de aprendizagem dos estudantes e é recomendado por poder trazer benefício aos mesmos e aos professores e, assim, contribuir para o aprimoramento do processo de ensino e aprendizagem.

\section{REFERÊNCIAS}

ALMEIDA, K. R. Descrição e análise de diferentes estilos de aprendizagem. Revista Interlocução, Belo Horizonte (MG), v. 3, n. 3, p. 38-49, 2010.

ALONSO, C.; GALLEGO, D.; HONEY, P. Los estilos de aprendizage: procedimientos de diagnóstico y mejora. 4. ed. Bilbao, Espanha: Ediciones Mensajero, 1999.

BECKER, P. Caracterização dos estilos e estratégias de aprendizagem dos estudantes do curso de farmácia da UFS. 2013. 116 f. Dissertação (Mestrado em Ciências da Saúde) Universidade Federal de Sergipe, Aracaju, 2013. Disponível em: https://ri.ufs.br/jspui/handle/riufs/3674. Acesso em: 20 maio 2020.

BIRRER, J. A.; MINELLO, I. F. Mapeamento dos estilos de aprendizagem de residentes de um programa multiprofissional da saúde. Imagens Da Educação, Maringá (PR), v. 6, n. 2, p. 19-28, 2016. Disponível em:

https://periodicos.uem.br/ojs/index.php/ImagensEduc/article/view/28186. Acesso em: 03 jun. 2020.

CARMO, B. B. T.; BARROSO, S. H. A.; ALBERTIN, M. R. Aprendizagem discente e estratégia docente: metodologias para maximizar o aprendizado no curso de engenharia de produção. Revista Produção Online, Piracicaba (SP), v. 10, n. 4, p. 779-817, 2010.

Disponível em: https://producaoonline.org.br/rpo/article/view/474. Acesso em: 03 jun. 2020.

CARVALHO, L. M. C. et al. Estilos de aprendizagem de estudantes universitários portugueses: uma proposta para visualização dos estilos predominantes. Revista Pensamento \& Realidade, São Paulo (SP), v. 34, n. 3, p. 3-20, 2019. Disponível em: https://revistas.pucsp.br/index.php/pensamentorealidade/article/view/46140. Acesso em: 17 jun. 2020.

CURY, H. N. Estilos de aprendizagem de alunos de engenharia. Páginas. In: CONGRESSO BRASILEIRO DE ENSINO DE ENGENHARIA, 27., 2000, Ouro Preto. Anais [...]. Ouro Preto, MG, 2000. Disponível em: https://faculdadebarretos.com.br/wpcontent/uploads/2015/11/ESTILOS-DE-APRENDIZAGEM-ALUNOS-ENG.pdf. Acesso em: 20 jun. 2020.

DA SILVA, D. M. O impacto dos estilos de aprendizagem no ensino de contabilidade da FEA-RP/ USP. 2006. 169 f. Dissertação (Mestrado em Controladoria e Contabilidade) Faculdade de Economia, Administração e Contabilidade de Ribeirão Preto, Universidade de

RPGE- Revista on line de Política e Gestão Educacional, Araraquara, v. 25, n. 2, p. 1544-1557, maio/ago. 2021. e-ISSN: 1519-9029 
São Paulo, Ribeirão Preto, 2006. Disponível em:

https://www.teses.usp.br/teses/disponiveis/96/96133/tde-24012007-152550/pt-br.php. Acesso em: 15 jun. 2020.

DE JESUS, E. M. S. et al. Metodologias de ensino e os estilos de aprendizagem na graduação em farmácia: um estudo piloto. Revista on Line de Política e Gestão Educacional, Araraquara (SP), v. 21, n. esp. 1, p. 621-639, 2017. Disponível em:

https://periodicos.fclar.unesp.br/rpge/article/view/9921. Acesso em: 20 maio 2020.

DE SOUZA L. M.; AVELINO, B. C.; TAKAMATSU, R. T. Estilos de aprendizagem e influência no processo de ensino-aprendizagem: análise empírica na visão de estudantes de Contabilidade. Revista Ambiente Contábil, Natal (PA), v. 9. n. 2, p. 379-400, 2017.

Disponível em: https://ojs.ccsa.ufrn.br/index.php/contabil/article/view/829. Acesso em: 25 fev. 2020.

FELDER, R. M.; FELDER, G. N.; DIETZ, E. J. The effects of personality type on engineering student performance and attitudes. Journal of Engeneering Education, v. 91, n. 1, p. 3-17, 2002. Disponível em: https://www.engr.ncsu.edu/wpcontent/uploads/drive/1QuEhY83i7u_3nZQmXOh31BoXOHnjLFa0/2002-longmbti.pdf. Acesso em: 30 maio 2020.

FELDER, R. M.; SILVERMAN, L. K. Learning and teaching styles in engineering education. Engineering Education, v. 78, n. 7, p. 674-681, 1988. Disponível em: https://www.engr.ncsu.edu/wp-content/uploads/drive/1QP6kBI1iQmpQbTXL08HS10PwJ5BYnZW/1988-LS-plus-note.pdf. Acesso em: 05 dez. 2019.

FELDER, R. M.; SOLOMAN, B. A. Index of Learning Styles (ILS). 1991. Disponível em: http://www.ncsu.edu/felder-public/ILSpage.html. Acesso em: 30 maio 2020.

FELDER, R. M.; SPURLIN, J. Applications, reliability and validity of the index of learning styles. International Journal of Engineering Education, v. 21, n. 1, p. 103-112, 2005.

Disponível em: https:/www.engr.ncsu.edu/wpcontent/uploads/drive/1ZbL_vMB7JmHGABSgr-xCCP2z-xiS_bBp/2005ILS_Validation(IJEE).pdf. Acesso em: 25 fev. 2020.

FRITSCH, E. F.; FLORES, C.; GIRAFFA, L. M. M. Estratégias de ensino. 2008. Disponível em: http://penta.ufrgs.br/ julio/tutores/estrateg.htm. Acesso em: 19 maio 2019.

GUIMARÃES, S. E. R.; BORUCHOVITCH, E. O estilo motivacional do professor e a motivação intrínseca dos estudantes: uma perspectiva da teoria da autodeterminação.

Psicologia: Reflexão e Crítica, Porto Alegre (RS), v. 17, n. 2, p. 143-150, 2004. Disponível em: https://www.scielo.br/j/prc/a/DwSBb6xK4RknMzkf5qqpZ6Q/?lang=pt. Acesso em: 12 jul. 2019.

HISFORD, C. C.; SIDERS, W. A. Felder-Soloman's index of learning styles: internal consistency, temporal stability, and factor structure. Teaching and Learning in Medicine, v. 22, n. 4, p. 298-303, 2010. Disponível em: https://pubmed.ncbi.nlm.nih.gov/20936578/. Acesso em: 12 jul. 2019. 
HUGHES, J. M. et al. Learning styles of orthodontic residents. Journal of Dental Education, v. 73, n. 3, p. 319-327, 2009.

KU, D. T.; SHEN, C. Y. Reliability, validity, and investigation of the index of learning styles in a Chinese language version for late adolescents of Taiwanese. Adolescence, v. 44, n. 176, p. 827-850, 2009.

KURI, N. P. Tipos de personalidade e estilos de aprendizagem: proposições para o ensino de engenharia. 2004. 337 f. Tese (Doutorado em Ciências Exatas e da Terra) - Universidade Federal de São Carlos, São Carlos, 2004. Disponível em: https://repositorio.ufscar.br/handle/ufscar/3332?show=full. Acesso em: 20 abr. 2019.

LIMA, A. I. A. O. Estilos de aprendizagem segundo os postulados de David Kolb: uma experiência no curso de odontologia da Unoeste. 2007. 91 f. Dissertação (Mestrado em Ciências Humanas) - Umiversidade do Oeste Paulista, Presidente Prudente, 2007. Disponível em: http://bdtd.unoeste.br:8080/jspui/handle/tede/845. Acesso em: 27 set. 2019.

LOPES, W. M. G. ILS: Inventário de estilos de aprendizagem de Felder-Soloman: identificação de sua validade em estudantes universitários de Belo Horizonte. 2002. 85 f. Dissertação (Mestrado em Engenharia de Produção) - Universidade Federal de Santa Catarina, Centro Tecnológico, Florianópolis, 2002. Disponível em: https://repositorio.ufsc.br/xmlui/handle/123456789/82278. Acesso em: 20 maio 2019.

MENDES DA SILVA, D.; DUTRA DE OLIVEIRA NETO, J. O impacto dos estilos de aprendizagem no ensino de contabilidade. Contabilidade Vista \& Revista, Belo Horizonte (MG), v. 21, n. 4, p. 123-156, 2010. Disponível em:

https://revistas.face.ufmg.br/index.php/contabilidadevistaerevista/article/view/810. Acesso em: 20 mai. 2019.

MURTA, S. G. Aplicações do treinamento em habilidades sociais: análise da produção nacional. Psicologia: Reflexão e Crítica, Porto Alegre (RS), v. 18, n. 2, p. 283-291, 2005. Disponível em: https://www.redalyc.org/articulo.oa?id=18818217. Acesso em: 03 jul. 2019.

OMAR, E. Perceptions of teaching methods for preclinical oral surgery: a comparison with learning styles. The Open Dentistry Journal, v. 11, p. 109-119, 2017. Disponível em: https://opendentistryjournal.com/VOLUME/11/PAGE/109/. Acesso em: 03 jul. 2019.

SADLER-SMITH, E. Learning styles: framework and instruments. Educational Psychology, v. 17, p. 51-63, 1997.

SCHMITT, C. S.; DOMINGUES, M. J. C. S. Estilos de aprendizagem: um estudo comparativo. Avaliação, Campinas (SP), v. 21, n. 2, p. 361-386, 2016. Disponível em: https://www.scielo.br/j/aval/a/CgyjHL3TRXbgwRdWphLbcks/?lang=pt. Acesso em: 30 maio 2020 .

SILVA, A. B. et al. Dimensões de um sistema de aprendizagem em ação para o ensino em Gestão. Administração: Ensino e Pesquisa, Rio de Janeiro (RJ), v. 13, n. 1, p. 11-46, 2012. Disponível em: https://raep.emnuvens.com.br/raep/article/view/97. Acesso em: 07 jul. 2019. 
VIOLA, S. et al. Analysis of felder-silverman index of learning styles by a data-driven statistical approach. In: IEEE INTERNATIONAL SYMPOSIUM ON MULTIMEDIA (ISM'06), 8., 2006, San Diego. Proceedings [...]. San Diego, Estados Unidos da América, 2006. p. 959-964. Disponível em: https://ieeexplore.ieee.org/document/4061286. Acesso em: 15 jun. 2020.

WOOD, J. T. Gendered lives: communication, gender, and culture. 1995. Disponível em: http://www.gbv.de/dms/ilmenau/toc/346407346.PDF. Acesso em: 15 jun. 2020.

\section{Como referenciar este artigo}

KUBLITSKI, P. M. O.; TOMAZINHO, P. H.; TOMAZINHO, F. S. F.; BRANCHER, J. A.; LEONARDI, D. P.; GABARDO, M. C. L. Estilos de aprendizagem dos estudantes da disciplina de endodontia de uma universidade privada no sul do Brasil. Revista on line de Política e Gestão Educacional, Araraquara, v. 25, n. 2, p. 1544-1557, maio/ago. 2021. eISSN:1519-9029. DOI: https://doi.org/10.22633/rpge.v25i2.14698

Submetido em: $29 / 01 / 2021$

Revisões requeridas em: 28/05/2021

Aprovado em: 15/06/2021

Publicado em: 01/08/2021 